\title{
Carnets
}

Revue électronique d'études françaises de l'APEF

Deuxième série - 6 | 2016

Exotopies de Barthes

\section{Barthes et le lexique de la critique}

\section{Alain Trouvé}

\section{OpenEdition}

\section{Journals}

Édition électronique

URL : http://journals.openedition.org/carnets/785

DOI : $10.4000 /$ carnets. 785

ISSN : 1646-7698

Éditeur

APEF

\section{Référence électronique}

Alain Trouvé, «Barthes et le lexique de la critique », Carnets [En ligne], Deuxième série - 6 | 2016, mis en ligne le 31 janvier 2016, consulté le 19 avril 2019. URL : http://journals.openedition.org/carnets/785 ; DOI : $10.4000 /$ carnets.785

Ce document a été généré automatiquement le 19 avril 2019.

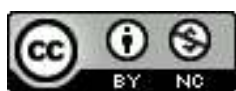

Carnets est mis à disposition selon les termes de la licence Creative Commons - Atribution - Pas d'utilisation commerciale 4.0 International. 


\title{
Barthes et le lexique de la critique
}

\author{
Alain Trouvé
}

\section{Barthes et le lexique de la critique : à propos de l'article « (Théorie du) texte »}

1 La préoccupation lexicale est ancienne dans l'œuvre de Barthes et remonte à sa rencontre dans les années 1950 avec Greimas, puis Georges Matoré, et à un projet de thèse en lexicologie structurale déposé en 1952, projet jamais mené à terme ${ }^{1}$. Cette prédilection pour le lexique va néanmoins irriguer toute l'écriture critique. On la retrouve sous la forme de nomenclatures qui, en lieu et place d'un plan raisonné, vont structurer de nombreux ouvrages, du Michelet (1954) aux Fragments d'un discours amoureux (1977), en passant par S/Z (1970), L'Empire des signes (1970) ou le Roland Barthes par Roland Barthes (1975), participant d'une recherche délibérée d'un étoilement du sens. Paradoxe d'une pensée hantée par le mouvement, le nom, gage en soi de stabilité, est toujours associé à des réseaux qui en font vaciller ou en complexifient le signifié, conformément au commentaire qui accompagne le projet de thèse initial: «mon répertoire (...) devrait permettre de voir le degré de mobilité d'articulation du lexique $»^{2}$. Cette mobilité lexicale est aussi le propre de la création littéraire. En ce sens, un cours donnés à l'EHESS en 1973-1974 et lisible depuis sa publication en 2010 s'intitule «Le lexique de l'auteur » (Barthes, 2010).

2 Le lexique de la critique, symétriquement, vise essentiellement le commentaire de l'œuvre d'autrui. Que dit Barthes lui-même sur ce mot «critique »? Dans un article de 1959 intitulé "Voies nouvelles de la critique littéraire en France», il distingue une "critique de lancée », (celle qui paraît dans les journaux et revues), critique porteuse d'un jugement, d'une évaluation et une « critique de structure » qui rattache l'œuvre à « un au-delà d'elle-même » (" Histoire ou Psyché »). Cette seconde critique est exposée à un double risque : ou bien la critique rend compte de l'Histoire, mais en idéologisant l'œuvre, elle l'irréalise ; ou bien elle rend compte de l'œuvre [sa dimension imaginaire], mais en la substantifiant, elle irréalise l'Histoire. ${ }^{3}$ La conclusion de l'article souligne l'enjeu de la résolution de ce dilemme, appelant de ses vœux une « synthèse » qui puisse 
répondre « aux tâches actuelles de l'explication scientifique ». L'horizon de cette critique est une théorie de la littérature, comme l'indique la question posée en ouverture du même article : "Qu'est-ce au fond que la littérature?»

3 Par cette réflexivité interrogative, la critique prend un sens philosophique, rejoignant la double visée cognitive et discriminante du criticisme kantien condensée dans la question : "Que puis-je savoir?». Réfléchir sur le lexique de la critique revient donc à examiner l'ambition de Barthes en matière de théorie littéraire, une ambition qui passe par la production de mots à valeur conceptuelle ou notionnelle. Quel est aujourd'hui le résidu actif de ce lexique revisité et élargi par Barthes?

L'invention conceptuelle peut se traduire de deux façons : par le néologisme de sens ou le néologisme de forme, plus spectaculaire, dont relèvent avec plus ou moins d'efficacité des créations comme "l'écrivant ", couplé avec l'écrivain, ou encore le "biographème ", promis à un assez bel avenir dans les études universitaires, voire le «scriptible » opposé au « lisible».

5 Avec le mot «texte » retenu ici, la création conceptuelle correspond plutôt à un réaménagement du sens. L'idée de "texte » occupe une place stratégique à la fois dans l'écriture critique de Barthes et dans l'impact universitaire de son œuvre. Il lui a donné une formulation à visée scientifique dans un article, « (Théorie du) texte ", rédigé en 1973 pour l'Encyclopédie Universalis. Nous-même prendrons pour modèle d'exposition cet article et non le Barthes de $S / Z$. Ce qui revient à entériner le clivage entre un langage de communication, celui d'un colloque, et un langage censé mimer le processus infini d'engendrement du sens.

6 On ira ainsi de la théorie à l'article proprement dit, puis à ses correctifs en forme de contrepoints, liés à d'autres contextes éditoriaux, l'ensemble traçant les contours d'une pensée de la littérature toujours active.

\section{Lexique et théorie}

\section{Le concept, seuil discriminant entre littérature et philosophie}

7 Selon un point de vue philosophique assez généralement admis depuis Kant, le concept est un « objet de la pensée (idée), correspondant à une règle ou schème lui assurant une valeur générale et abstraite» (Rey, 2009 : 387). Cette pensée se nomme aussi «faculté de juger déterminante» dans la Critique de la raison pure. Dans sa troisième Critique, Kant (1995) la distingue de la "faculté de juger réfléchissante ", d'où procède, entre autres, le jugement esthétique mettant en jeu le sujet. Autrement dit, il n'y a de concept généralisant que de l'objet, et si l'on veut aborder l'activité du sujet, il convient d'utiliser un autre mot.

8 Jean-Marie Schaeffer développe de façon similaire cette idée. Il oppose la connaissance scientifique (conceptuelle) qui subsume le particulier sous la catégorie du général et la connaissance esthétique, activité associative horizontale :

On dira plutôt que c'est la différence entre activité cognitive horizontale (associative) d'un côté, activité cognitive verticale (généralisante ou particularisante) de l'autre, qui distingue la relation cognitive de niveau 1 [incluant la conduite esthétique] de la relation de niveau 2 [scientifique]. (Schaeffer, 1995 : 165-166) 
Ce point de vue semble partagé par les poètes lorsqu'ils s'adonnent à la réflexivité. On peut en effet rapprocher le concept et le signifié dénoté ou définition des dictionnaires. Pour Yves Bonnefoy, par exemple, la poésie prend en défaut l'usage catégoriel du langage : J'appelle poésie ce qui, dans l'espace des mots, notre monde, a mémoire du surcroît de ce qui est sur ses représentations : mémoire des référents dans l'espace des signifiés. (Bonnefoy, $2002:$ 8) On se souvient aussi de la critique adressée par Mallarmé au langage de communication, "l'universel reportage». Sous une forme un peu plus polémique, Barthes dénonce dans un entretien de mai 1970 à propos de ses deux livres, S/ $\mathrm{Z}$ et L'Empire des signes le « retour du signifié, de la théologie, du monothéisme, de la loi » (Barthes, 1970c : 668).

10 Pourtant, le glissement du sens scientifico-philosophique au sens lexical ne laisse pas de poser problème. Il supposerait que le sens d'un mot dans son usage de communication socialisée soit ramené à une seule définition. Or le signifié d'un mot est souvent ramifié en branches. C'est pourtant cette assimilation que semble reprendre Barthes quand il place l'usage communicationnel du langage sous le signe du paradigme pour lui opposer "le neutre» (Barthes, 2002). Le neutre selon Barthes est « ce qui déjoue le paradigme», mais en organisant un cours entier sur le neutre, il crée encore un paradigme... Une critique similaire avait été adressée à Derrida à propos de la déconstruction, terme destiné à montrer la déstabilisation potentielle de toute signification, mais qui en tant que terme fonde encore une catégorie linguistique.

11 L'une des réponses adressées à cette critique du concept a été donnée par Nietzsche qui a rapproché la philosophie du mythe et de la métaphore, empruntant à la littérature certaines de ses modalités expressives. Nietzsche, précisément, figure en bonne place dans le Panthéon de Barthes.

\section{La stratégie (autocritique) de Barthes et le lexique}

12 «La science est grossière, la vie est subtile, et c'est pour corriger cette distance que la littérature nous importe", affirme Barthes dans la Leçon inaugurale de 1977 (Barthes, 1978h : 434). Comprenons : la critique et la philosophie devraient pencher vers l'art pour se rapprocher de la vérité. En tant que critique, Barthes n'a pourtant pas renoncé à toute ambition de scientificité. «Science » est d'ailleurs statistiquement un mot assez présent dans son discours. Il s'agit plutôt de refonder cette ambition par une écriture nouvelle.

Deux caractéristiques de cette écriture tentent de concilier la rigueur de la science et la souplesse des lettres : la forme répertoire et l'échelonnement de la parole.

14 Toute parole critique est suspecte puisqu'elle porte en elle les marques de sa méconnaissance, historique et psychique. En adoptant la forme du répertoire, en multipliant les entrées, Barthes veut déjouer l'illusion d'un sujet maître du sens et montrer au contraire l'engendrement potentiel, à l'infini, du sens. C'est encore la méthode qui prévaut dans $S / Z$ qui programme la progression dans la lecture de la nouvelle Sarrazine à partir de micro-unités textuelles et d'entrées lexicales non hiérarchisées : «XVI. La beauté »; «XVII. Le camp de la castration »; « XVIII. Postérité du castrat »; « XIX. L'indice, le signe ; l'argent ». Barthes précise :

Méthode de lecture qui amène à la pluralisation de la critique, à l'analyse structurale du récit, à la science du texte, à la fissuration du savoir dissertatif, l'ensemble de ces activités prenant place dans l'édification (collective) d'une théorie libératoire du Signifiant » (S/Z, 1970, Quatrième de couverture) 

objectivité, il s'agit d'assigner toute parole à son imaginaire en rendant le propos à son cadre énonciatif et en reconnaissant derrière les considérations savantes le désir du sujet parlant. On est frappé ainsi dans le cours La Préparation du roman (2003) par la place qu'occupe dans l'ensemble une rubrique comme le « désir de roman ", avec tout ce qu'elle comporte d'implication personnelle. Dans cette forme limite qu'est le Roland Barthes par Roland Barthes, forme limite puisque l'écriture essayiste semble laisser place au projet autobiographique et donc à une écriture littéraire, Barthes conserve quelque chose de sa méthode scientifique. Il tente de prendre à revers toute la tradition en posant la noncoïncidence du sujet écrit et du sujet écrivant, comme le suggère le titre dont la forme syntaxique marque la distinction entre l'objet et l'agent ${ }^{4}$.

\section{Le rôle du cadre énonciatif}

1. On remarque aussi une certaine hétérogénéité du cadre institutionnel ou éditorial au sein duquel se déploie la parole. Quatre catégories peuvent être grossièrement distinguées

2. Des articles sont publiés dans de très nombreuses revues représentant un large éventail du paysage idéologique. Certaines sont des revues d'avant-garde. Paradoxe : la parole dans une revue comme Communication s'attache à ruiner l'idée d'un langage de communication.

3. Les essais, le plus souvent publiés aux éditions du Seuil, qui s'intéressent aux travaux novateurs, jouent le jeu d'une certaine dissémination du sens afin de contourner la fausse objectivité des sciences humaines.

4. Les conférences et séminaires à l'EHESS puis au Collège de France sont affectés d'une ambivalence similaire : ces lieux mixtes, de savoir et de prestige, sont aussi des lieux de contre-pouvoir. La pensée critique qui s'y affirme va devenir néanmoins l'inspiratrice de nombreux travaux universitaires et fera ainsi retour dans le champ institutionnel.

5. Restent les lieux dépositaires d'un savoir scientifique à vocation large (universelle) que sont les encyclopédies. La commande implicite appelle l'exposition articulée (ordonnée selon un raisonnement) d'un contenu notionnel. Barthes s'y est adonné à quelques reprises, parmi lesquelles figurent notamment l'article « Ecoute » (Encyclopédie Einaudi, 19775) et l'article qui nous intéresse.

\section{« (Théorie du) texte » : forme et contenu, intérêt et difficultés}

Dans l'article écrit pour l'Encyclopédie Universalis en $1973^{6}$, la parenthèse " (Théorie du)» est importante : elle affirme une ambition que confirme aussi l'usage fréquent des mots « science » et « concept ».

\section{Le sujet de l'énonciation se veut pluriel}

Barthes s'appuie largement sur les travaux de Julia Kristeva et se réfère accessoirement à d'autres membres du groupe Tel Quel ${ }^{7}$. Celui qui rédige l'article est plus que le sujet Barthes, c'est le sujet collectif de la science (nouvelle). 


\section{Plan de l'article}

19 Le texte s'organise en quatre parties - 1/ La crise du signe, 2/ La théorie du texte, 3/ Le texte et l'œuvre, 4/ La pratique textuelle - quatre parties dont le mouvement relève d'une écriture raisonnée, presque dialectique et pas du tout tabulaire.

L'introduction annonce une critique de la conception traditionnelle et phénoménale du texte comme garantie de stabilité et de légalité du sens.

21 La « crise du signe » (1) s'attaque à la vision du texte comme dépositaire d'une Vérité. La démonstration prend appui sur Nietzsche, sur le Cercle de Prague pour la linguistique, sur le marxisme, pour l'Histoire, et sur la psychanalyse. L'instabilité du signe renvoie la parole à ce qui est l'Autre du sujet.

22 La «théorie du texte » (2) emprunte à Kristeva plusieurs concepts : le couple signifiance (sens en mouvement) - signification (sens fixe), le couple phéno-texte (l'objet texte dans sa matérialité) - géno-texte (le sens en gestation). Le géno-texte est «un domaine hétérogène, à la fois verbal et pulsionnel». Est également repris le concept d'intertextualité forgé par la même Kristeva dans Séméiotikè (1969). Le texte n'est plus voile du sens mais tissu d'énoncés venant d'horizons divers.

23 «Le texte et l'œuvre » (3) oppose l'œuvre «qui se tient dans la main » au texte situé « dans le langage ». L'une (l'œuvre) serait hétérogène aux pratiques sociales et éditoriales liées à la production de l'objet livre, l'autre (le texte) « homogène au langage ». Le texte est conçu dans son acception la plus large. Barthes intègre en effet dans la pratique textuelle la lecture, "nouvel objet épistémologique». Il y inclut aussi des pratiques signifiantes non verbales: « La théorie du texte tend à abolir la séparation des genres et des arts » (1973e : 455).

24 «La pratique textuelle »(4) ou analyse textuelle semble se situer encore du côté du lecteur : elle emprunte à Séméiotikè un dernier concept, celui de sémanalyse, dont l'objet est le «recoupement du phéno-texte et du géno-texte» nommé «à la suite des postformalistes russes" et de Kristeva un "idéologème», "concept qui permet d'articuler le texte sur l'intertexte et de 'le penser dans les textes de la société et de l'histoire'" (ibid. : 457). Le devenir de cette théorie implique "que le commentaire soit luimême un texte» (ibid. : 457); mais "la seule pratique que fonde la théorie du texte est le texte lui-même» (ibid. : 457). Cette seconde proposition réunit l'écrivain et le lecteur dans une seule et même entité, le texte, dont la pratique, assortie d'un évanouissement des frontières du sujet, serait garante de «jouissance »: on aura reconnu l'allusion au Plaisir $d u$ texte, très proche chronologiquement de la rédaction de cet article et seulement cité dans la bibliographie.

L'article présente donc une structure logique et réflexive. La première partie pose les fondements de la seconde, cœur de la définition, sur le mode de la causalité explicative. Les parties 3 et 4 tirent des conséquences de cette définition de façon actuelle (3) et prospective (4). La partie 4 se veut de surcroît une sorte de dépassement dialectique de la troisième. $3:$ «Le texte est un concept scientifique (...) et en même temps une valeur critique » (ibid.: 455); $4:$ «l'analyse textuelle tend à substituer [à une science positive] une science critique » (ibid. : 456). Cohérence et unité de la pensée barthesienne : on n'est pas loin des deux sens de la critique posés dans l'article de 1959. 


\section{Point clefs et problèmes}

\section{Ambiguiités et contradictions}

31 Sur la question du statut conceptuel du texte, on relève la présence de propositions contradictoires en lien avec le contexte éditorial. Un article de 1972, presque contemporain de celui donné à l'Encyclopédie Universalis et paru dans la revue Communications 8 , propose l'énoncé suivant: «Suivons un instant d'article en article la main commune qui, loin d'écrire la définition du Texte (il n'y en a pas : le Texte n'est pas 
un concept), décrit (dé-script) la pratique d'écriture. » (130-131). De son côté, l'article de 1973 dira : « le texte est un concept scientifique » (1973e : 453). Pas d'évolution explicable par une maturation temporelle, d'un énoncé à l'autre, mais une inflexion liée au contexte énonciatif. Pour une encyclopédie, on ne peut décrire un article...

L'acception intensive du texte est quasiment intenable: Barthes lui-même ne s'y tient pas, y compris dans son article : le syntagme amusant du texte «textuel» le dit à sa façon. Les lecteurs de Barthes oublieront ce sens intensif et prendront ou reprendront le texte dans son sens phénoménologique ( = l'écrit manuscrit ou imprimé que je tiens dans la main) mais continueront à lui associer certaines des propriétés évoquées dans l'article. Il en va ainsi de la convertibilité des systèmes sémiotiques (image et texte) et de la confusion des écritures dans le texte commun.

La prépondérance de la sémiologie sur la sémiotique tend à homogénéiser les pratiques signifiantes ${ }^{9}$. Les grandes analyses sémiologiques de Barthes sur les mythes et la publicité n'ont pas peu contribué à instaurer ce glissement. La question est celle du statut des pratiques signifiantes, ouvertes ou non à de l'hétérogène. L'article de l'EU fait place à cette idée à deux reprises.

Le géno-texte emprunté à Kristeva est analysé comme « un domaine hétérogène, à la fois verbal et pulsionnel [je souligne]» (ibid.: 450). On est bien ici dans une approche sémiotique. Dans l'opposition entre l'œuvre et le texte, il était dit que « L'œuvre peut être définie en termes hétérogènes au langage (format, déterminations socio-historiques) » ( ibid. : 452), contrairement au texte. La tension entre homogène et hétérogène se réduit avec la prépondérance du modèle sémiologique. La théorie de l'image comme texte aboutit à des expressions courantes dans les études universitaires comme «intertexte pictural», expressions qu'il conviendrait d'interroger. Surcroît de difficulté: Barthes rapporte l'hétérogénéité de l'œuvre aux « textes de la société et de l'histoire » (ibid. : 457). Il assimile ainsi le texte au sens phénoménologique et le code au sens large (ensemble des règles, lois, coutumes œuvrant dans l'implicite de la vie sociale et dépassant le cadre de textes législatifs déterminés). Prise dans sa globalité, la pensée de Barthes n'élimine pas la contradiction entre l'hétérogène et l'homogène sans doute nécessaire pour penser le rapport texte/image.

35 Il en va de même s'agissant de la confusion des niveaux de discours qui aboutit à l'affirmation selon laquelle tout est texte, rien que texte. Auteur et lecteur seraient ainsi des rôles interchangeables. Revenons à la phrase déjà commentée: «Le texte reste de part en part homogène au langage : il n'est que langage et ne peut exister qu'à travers un autre langage [je souligne]» (ibid.: 452). La conséquence recèle une idée féconde, celle d'une lecture créatrice et d'un tiers lecteur, distinct par son langage du sujet auteur ${ }^{10}$. Mais cette reconnaissance reste problématique dès lors que les deux sont invités plus loin à se rejoindre dans un travail sur le signe qui abolit les frontières entre sujets sous le signe de la jouissance.

\section{Barthes corrigé ou complété par lui-même}

Une lecture élargie de Barthes tend à rectifier le côté abrupt de certaines formulations. S'expliquant dans un journal culturel comme Les Lettres françaises, destiné à un large public, Barthes propose une distinction contraire à l'unification des discours. À propos de son livre $S / Z$ qui instituait à partir de la nouvelle de Balzac Sarrasine un nouveau mode de lecture, il situe son discours par rapport à un " texte tuteur $»^{11}$. Cette précision verbale 
très intéressante entérine la distinction entre texte d'écrivain et texte de lecteur. Autrement dit, il y a du métalangage. Dans son cours La Préparation du roman, Barthes pose encore le roman comme ce qui échappe au métalangage, ce qui revient à admettre le statut de métalangage de certains énoncés. Il note en ce sens : «il y a une poussée à l'indifférenciation des écrits, freinée par les catégories éditoriales » (Barthes, 2003 : 201).

econnaissance de deux niveaux ou modalités du discours se vérifie aussi par le désir de conversion qui pousse dans les dernières années Barthes à se projeter dans l'écriture de roman comme forme spécifique, curieusement articulée au Haïku, on va y revenir.

Deux livres admettent implicitement et conjointement l'existence du hors-texte et du métalangage : L'Empire des signes (1970) et La Chambre claire (1980) ${ }^{12}$.

39 L'Empire des signes répond à une commande de l'éditeur suisse Skira invitant des artistes ou de grands spécialistes de l'art à dire comment ils conçoivent la création. L'édition prévoit de faire jouer le texte et l'image, chacun des contributeurs les associant à sa manière. Au moment de la rédaction du livre, Barthes ne parle pas la langue japonaise qu'il considère « comme une langue inconnue » (Barthes, 1970b : 352). Mais il intègre tout ce qu'il voit et perçoit du Japon - visages, configuration urbaine, cuisine, danse ou pratique sportive - dans un système de signes. Il dit de lui à la troisième personne que « le Japon l'a mis en situation d'écrire » (ibid.). Il décrit et analyse un repas japonais comme système signifiant. En un sens, L'Empire des signes relève encore d'une sémiologie omnipotente. Mais la convertibilité des systèmes symboliques trouve sa limite dans l'ancrage territorial qui leur assigne une part d'hétérogénéité : "Ce qui peut être visé dans la considération de l'Orient (...) c'est la possibilité d'une différence, d'une mutation, d'une révolution dans la propriété des systèmes symboliques » (ibid. : 351). Un en-deçà du texte, nous dirions volontiers un arrière-texte ${ }^{13}$, vient en relativiser l'universelle traductibilité.

Barthes intègre à son livre une réflexion sur le haïku, version condensée du haïkaï relié à son soubassement Zen : «tout le Zen dont le haïkaï n'est que la branche littéraire, apparaît comme une immense pratique destinée à arrêter le langage »(Barthes, 1970b : 408), comprenons : le sens. Il ne s'agit plus de mobilité comme dans la signifiance, mais de vide. Dans l'interview déjà citée, Barthes déclare avoir ainsi découvert «l'éthique du signe vide $»^{14}$. Ce vide renvoie à la confrontation à un référent hors-langage de deux façons : 1/ en tant que produit de la culture zen, il est adossé à un espace géoculturel ; 2/ les haïkus admirés, quant à eux, se contentent d'enregistrer les micro-événements d'une présence au monde par l'articulation élémentaire de quelques perceptions: "Avec un taureau à bord / Un petit bateau traverse la rivière, / À travers la pluie du soir » (Barthes, 1970b : 102). Barthes voit le haïku «articulé sur une métaphysique sans sujet et sans dieu » (ibid. : 410), disons un sujet minimal, s'effaçant devant la contemplation de ce qui est posé en face du langage comme l'extériorité du monde: «C'est cela, c'est ainsi, dit le haïku, c'est tel » (ibid. : 415).

41 Le « c'est cela » peut être relié au "ça a été ", dont l'idée surgit de la contemplation de certaines photographies. La Chambre claire fonde la dialectique du punctum et du studium. Si le studium renvoie au savoir culturel, donc aux langages institués susceptibles de décoder l'image, le punctum saisit celui qui regarde la photographie en le renvoyant à une sorte de hors-texte existentiel qui le concerne de près ou de loin. S'opère ainsi un certain 
retour de la phénoménologie contre la Science que manifeste aussi la recherche du « noème de la photo » évoqué dans La Préparation du roman (Barthes, 2003 : 114).

\section{Faire œuvre littéraire}

\section{BIBLIOGRAPHIE}

BARTHES, Roland (1959a). « Voies nouvelles de la critique en France ». (2002), Cuvres complètes I.

Paris : Seuil.

BARTHES, Roland (1970b). L'Empire des signes. (2002), Cuuvres complètes III. Paris : Seuil.

BARTHES, Roland (1970c). « Entretien avec Raymond Bellour ». (2002), CEuvres complètes III. Paris :

Seuil.

BARTHES, Roland (1972d). « Jeunes chercheurs ». (2002), Euvres complètes IV. Paris : Seuil. 
BARTHES, Roland (1973e). « (Théorie du) texte ». (2002), CEuvres complètes IV. Paris : Seuil.

BARTHES, Roland (1975f). « Barthes puissance trois ». (2002), CEuvres complètes IV. Paris : Seuil.

BARTHES, Roland (1977g). « Écoute ». (2002), Euvres complètes IV. Paris : Seuil.

BARTHES, Roland (1978h). « Leçon ». (2002), Euvres complètes V. Paris : Seuil.

BARTHES, Roland (2002). Le Neutre, cours au collège de France, 1977-1978. Paris : Seuil.

BARTHES, Roland (2003). La Préparation du roman I et II, cours et séminaires au Collège de France,

1978-1980. Paris : Seuil.

BARTHES, Roland (2010). Le Lexique de l'auteur, Séminaire à l'École pratique des hautes études 1973-1974.

Paris : Seuil.

BONNEFoY, Yves (2002). Sous L'Horizon du langage. Paris : Mercure de France.

BRANDOM, Robert ([2000] 2009). L'Articulation des raisons, Introduction à l'inférentialisme, trad.

Claudine Tiercelin et Jean-Pierre Cometti. Paris : CERF.

DERRIDA, Jacques (1967). De la Grammatologie. Paris : Minuit.

GLADIEU, Marie-Madeleine, Pottier, Jean-Michel et Trouve, Alain (2013). L'Arrière-texte Pour repenser le littéraire. Bruxelles : Peter Lang.

KANT, Emmanuel ([1750] 1995). Critique de la faculté de juger, traduction inédite d'Alain Renaut.

Paris : Aubier.

REY, Alain (dir.) (2009). Le Grand Robert de la langue française, deuxième édition, II. Paris : Le Robert, p. 387.

SAMOYAUlt, Tiphaine (2015). Roland Barthes. Paris : Le Seuil.

SCHAEFFER, Jean-Marie (1996). Les Célibataires de l'art. Paris : Gallimard.

TROUVE, Alain (2004). Le Roman de la lecture. Liège : Mardaga.

\section{NOTES}

1. Thèse sous la direction de Charles Bruneau et intitulée « Le Vocabulaire des rapports entre l'Etat, les patrons et les ouvriers de 1827 à 1834 d'après les textes législatifs, administratifs et académiques. " (Tiphaine Samoyault, $2015: 239$ ).

2. Dossier de carrière scientifique de Barthes, cité par Tiphaine Samoyault (2015:241).

3. Politica (Barthes, 1959a: 979), désormais : OC.

4. Dans le même esprit, le commentaire de ce livre donné par Barthes à Maurice Nadeau pour La Quinzaine littéraire, le $1^{\mathrm{er}}$ mars 1975, s'intitule « Barthes puissance trois » (1959c : 777).

5. Barthes (1978: 340-352).

6. Article repris dans Barthes (1973e : 443-459).

7. Dans la bibliographie, on trouve aussi les noms de Baudrillard, Derrida, Sollers, Todorov, entre autres, entourant les deux œuvres récentes de Barthes, $S / Z$ et Le Plaisir du texte.

8. "Jeunes chercheurs", Communications, $\mathrm{n}^{\circ} 19,1972$, article repris dans Barthes (1972d : 126-132).

9. Dans son cours La Préparation du roman, Barthes se reconnaît d'abord sémiologue: « Paradoxalement, quoi que, en principe, 'sémiologue', j'entends 'sémiotique' dans un sens très irrégulier, proprement nietzschéen (Ecce Homo) : il parle de son rapport à Schopenhauer, il les a institués comme signes de lui-même » (Barthes, 2003 : 192). 
10. Nous-même avons avancé l'idée que la lecture, au sens actif du terme, implique de la part du lecteur la production de son propre énoncé métatextuel ou texte de lecture (voir à ce sujet, Trouvé, 2004).

11. Entretien avec Raymond Bellour (Barthes, 1970c: 666).

12. Ouvrages repris respectivement dans les volumes III et V des Euvres complètes.

13. Voir à ce sujet, Marie-Madeleine Gladieu, Jean-Michel Pottier et Alain Trouvé (2013).

14. Entretien avec Rémi Bellour (Barthes, 1970c : 667).

\section{RÉSUMÉS}

Le lexique constitue dans l'œuvre de Barthes un point d'achoppement entre la prétention scientifique à l'objectivité et la mobilité du sens. Si Barthes a indéniablement enrichi le lexique de la critique, la contradiction est manifeste entre la conceptualisation catégorielle à l'œuvre dans l'article "(Théorie du) texte" rédigé pour l'Encyclopédie Universalis et la variabilité des acceptions liée aux contextes énonciatifs et socioculturels. La présente étude montre l'incidence contestable dans la critique universitaire d'un "pantextualisme », dérivé d'un usage abusif de l'intertextualité, notion centrale dans la « théorie du texte ». Le pantextualisme occulte la part de non-convertibilité entre texte et image ou la possible existence d'un hors-texte, alors même que la lecture du dernier Barthes corrige cette vision simplifiée. Il faut relire Barthes contre et avec lui.

In the works of Barthes, the vocabulary of criticism constitutes a stumbling block between the scientific pretention to the objectivity and the mobility of the meaning. While Barthes indeniably enriched the vocabulary of criticism, there is an obvious contradiction between the conceptualization in categories present in the article "(Theory of) the text ", written for the Encyclopédie Universalis and the variability of the meanings linked to the enunciative and sociocultural contexts. This study shows the debatable impact in the university criticism of a "pantextualism » derived from an inappropriate use of intertextuality, a central notion in the " (Theory of) the text ». The " pantextualism » masks the part of non-convertibility between text and image or the possible existence of an « hors-text », whereas the reading of the last works of Barthes corrects this simplified vision. It is necessary to re-read Barthes against and with him.

\section{INDEX}

Mots-clés : concept, notion, intertextualité, métalangage, contexte, image, hors-texte, science, roman

Keywords : concept, notion, intertextuality, metalanguage, context, image, hors-text, science, novel 


\section{AUTEUR}

\section{ALAIN TROUVÉ}

Universidade de Lisboa Université de Reims Champagne-Ardenne

alain.trouve@wanadoo.fr 